\title{
Neutrino mass, $0 v \beta \beta$ signature in doublet left-right symmetric theories and its cosmological implications
}

\section{Chayan Majumdar*}

Indian Institute of Technology Bombay, Mumbai 400076, India

E-mail: chayan@phy.iitb.ac.in

\section{Sudhanwa Patra}

Indian Institute of Technology Bhilai, Chhattisgarh 492015, India

E-mail: sudhanwaditbhilai.ac.in

\section{Supriya Senapati}

Indian Institute of Technology Bombay, Mumbai 400076, India

E-mail: supriya@phy.iitb.ac.in

\section{Urjit A. Yajnik}

Indian Institute of Technology Bombay, Mumbai 400076, India

E-mail: yajnik@phy.iitb.ac.in

\begin{abstract}
We consider a version of doublet Left-Right Symmetric Model (LRSM) in which the scalar sector consists of a Higgs bidoublet $(\Phi)$, Higgs doublets $\left(H_{L, R}\right)$ and a charged scalar $\left(\delta^{+}\right)$with $B-L=$ 2 leading to one-loop generated Majorana masses for neutrinos and thereby, leads to beyond Standard Model (BSM) contributions to neutrinoless double beta decay $(0 v \beta \beta)$. We embed such a novel framework in a non-SUSY $S O(10)$ GUT leading to successful gauge coupling unification for two different scenarios. Due to sizeable $W_{L}-W_{R}$ mixing to neutrinoless double beta decay, this model can saturate the present bound from GERDA and KamLAND-Zen experiments. Also, we briefly explain how keV-MeV range $\mathrm{RH}$ neutrino arising from our model can be considered as warm Dark Matter (DM) candidate to address various cosmological issues.
\end{abstract}

40th International Conference on High Energy physics - ICHEP2020

July 28 - August 6, 2020

Prague, Czech Republic (virtual meeting)

\footnotetext{
* Speaker.
} 


\section{Introduction}

Though numerous theoretical predictions of Standard Model (SM) match really well with the recent-day collider findings, some theoretical and observational inconsistencies still persist, seeking framework beyond SM (BSM). Left-Right Symmetric Theories (LRSMs) [1] are one of such scenarios where we can explain the origin and smallness of neutrinos as well as parity violation in low-scale weak phenomenology in a unified manner. In this work, we describe various phenomenological and cosmological implications of a doublet variant LRSM [2].

\section{Model and Phenomenology of our framework}

\subsection{Particle Content and Gauge Coupling Unification}

Unlike SM, all the fermions in LRSM framework are part of doublets. The gauge group of such framework corresponds to $S U(3)_{C} \otimes S U(2)_{L} \otimes S U(2)_{R} \otimes U(1)_{B-L}$. Left and Right-handed quark and lepton doublets constitute the fermion spectrum. In scalar sector, we have considered Higgs bidoublet $\Phi$ with $B-L=0$, Higgs doublets $H_{L, R}$ with $B-L=1$ and a singly charged scalar $\delta^{+}$with $B-L=2[3]$.

With this particle content, we have embedded such framework in a non-supersymmetric (nonSUSY) GUT scenario with unification and LR-symmetry breaking at around $10^{16} \mathrm{GeV}$ and $M_{R} \sim$ $10^{10} \mathrm{GeV}$ respectively. Such $M_{R}$ scale is far beyond the reach of recent-day collider searches.
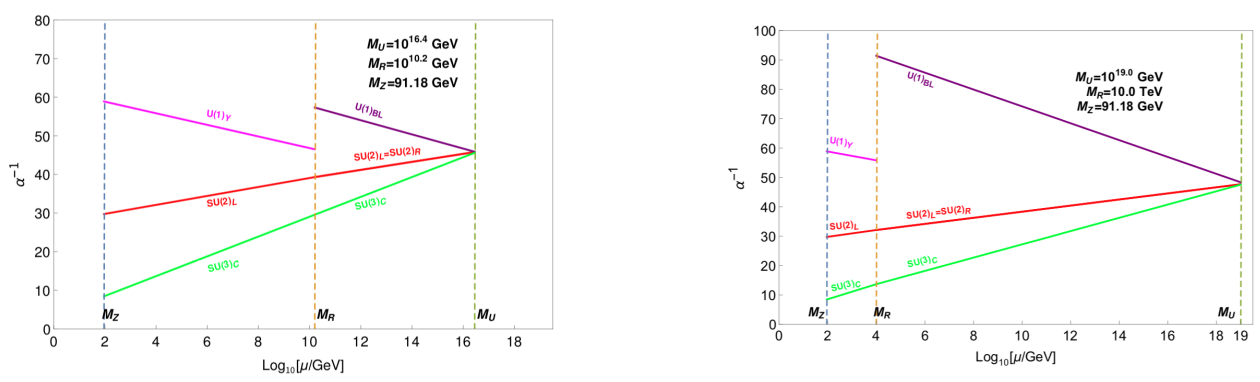

Figure 1: Left Panel : Gauge Coupling running with minimal particle spectrum, Right Panel : Low scale $M_{R}$ in context of extended LRSM.

To have a rich phenomenology in context of collider findings, we have extended our model beyond discussed LRSM scenario by including new particles $\xi(6,1,1,4 / 3)$ and another three copies of $\delta^{+}$. In this extended LRSM, $M_{R}$ scale has been brought down to few TeV leading to new Right gauge boson masses $M_{W_{R}}, M_{Z_{R}} \sim \mathscr{O}(\mathrm{TeV})$ which is much favorable than the previous case.

\subsection{Neutrino mass and $0 v \beta \beta$ signature}

Spontaneous Symmetry breaking (SSB) of such framework has been carried out by the vacuum expectation values (VEVs) acquired by various Higgs scalars, $\langle\Phi\rangle=\operatorname{diag}\left(v_{1}, v_{2}\right),\left\langle H_{L, R}\right\rangle=v_{L, R}$. Only with Higgs bidoublet and doublets we can generate Dirac masses for neutrinos and other fermions. To generate Majorana mass term (radiatively at one-loop order) for neutrinos, we have introduced $\delta^{+}$particle in the framework. Possible Feynman diagrams are given in left panel of Fig.2. Loop-induced Majorana mass term for neutral fermions can be written as, 


$$
M_{L, R}^{1-\text { loop }}=\frac{\lambda^{\prime}\left\langle H_{L}\right\rangle\left\langle H_{R}\right\rangle}{16 \pi^{2}} \frac{\lambda^{L, R} M_{l} Y_{l}^{T}}{M^{2}}\left(\frac{\log \left[\frac{M_{\ell}^{2}}{M_{\delta^{+}}^{2}}\right] M_{\delta^{+}}^{2}}{M_{\delta^{+}}^{2}-M_{\ell}^{2}}-\frac{\log \left[\frac{M_{\ell}^{2}}{M_{\Phi}^{2}}\right] M_{\Phi}^{2}}{M_{\Phi}^{2}-M_{\ell}^{2}}\right)
$$

with $M=\max \left(M_{\delta^{+}}, M_{\Phi}\right)$ and $\lambda^{\prime}, \lambda^{L, R}, Y_{l}$ are corresponding couplings. Resulting mass eigenvalues for light and heavy neutrinos are resembling with the type-I seesaw relations.
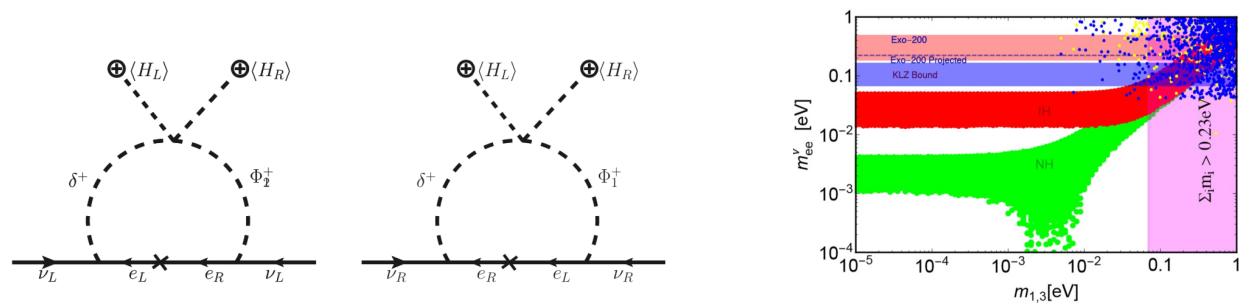

Figure 2: Left Panel : Radiative mass generation, Right Panel : BSM 0v $\beta \beta$ signatures within the model.

Neutrinoless double beta decay $(0 v \beta \beta)$ [4] describes a rare process involving the sponatneous decay of two neutrons to two protons and electrons with no accompanying external neutrinos. Any indication of observation of such process would claim the Majorana nature of neutrinos as well as lepton number violation (LNV) in physical processes. Within this framework, we can have significant $0 v \beta \beta$ signatures arising from purely left or right handed gauge boson mediation or mixed helicity $\lambda$ and $\eta$ diagrams. In the right panel of Fig.2 we have shown that for $M_{R} \sim$ few TeV, significant contributions are arising from $\lambda, \eta$ channels to satisfy KamLAND-Zen [5], GERDA [6] bounds.

\section{Connection to Cosmology}

With $M_{R} \sim$ few TeV and heavy neutrino masses around keV-MeV scale [7, 8], we can consider the lightest sterile neutrino to serve the role of warm dark matter (WDM) candidate to satisfy the correct relic density criteria for DM in universe. Such low-scale right handed neutrinos (RHNs) can create the overabundance problem which can be overcome by the out-of-equilibrium late entropy production mechanism with the corresponding decay channels of heavier RHNs to light relativistic SM particles. Such framework can easily saturate various cosmological and astrophysical bounds.

\section{References}

[1] Mohapatra R. N. and Pati J. C., Phys. Rev. D 11, 2558 (1975)

[2] Majumdar C., Patra S., Senapati S. and Yajnik U. A., Nucl. Phys. B951, 114875 (2020)

[3] Fileviez P., Murgui C. and Ohmer S., Phys. Rev. D 94, 051701 (2016)

[4] Mohapatra R. N. and Senjanović G., Phys. Rev. D 23165 (1981)

[5] Gando A., et. al, Phys. Rev. Lett. 110, 062502 (2013)

[6] Agostini M., et. al, Phys. Rev. Lett. 111, 122503 (2013)

[7] Bezrukov F., Hettmansperger H. and Lindner M., Phys. Rev. D 81, 085032 (2010)

[8] Nemevšek M. and Senjanović G. and Zhang Y., JCAP 1207, (2012), 006 